\title{
Additive Manufacturing Methods for Soft Magnetic Composites (SMCs)
}

Nicole C. Benack ${ }^{1}$, Tony Wang ${ }^{2}$, Kyle Matthews ${ }^{3}$, and Mitra L. Taheri ${ }^{4}$

${ }^{1 .}$ Drexel University, Materials Science and Engineering Department, Philadelphia, PA, USA.

2. Drexel University, Materials Science and Engineering Department, Philadelphia, PA, USA.

3. Drexel University, Materials Science and Engineering Department, Philadelphia, PA, USA.

4. Drexel University, Materials Science and Engineering Department, Philadelphia, PA, USA.

Currently, electromagnetic core designs are limited by silicon steel laminations, which are used to reduce eddy current losses. Steel laminations are difficult to shape into complex designs and create significant waste during processing. They are expensive to manufacture in complex shapes for novel motors which is why powder metals, more specifically soft magnetic composites (SMCs), are a promising alternative to laminations [1]. These composites are made from ferromagnetic powder particles, such as iron powder, and a thin insulating coating that separates each particle. The selection of iron-based powder, such as pure iron or an iron-nickel alloy, is based on whether AC or DC magnets are involved in the final assembly [1-2]. The coating on the SMC can be either an organic, or inorganic coating. SMC cores made from organic (polymer) bonded iron particles should have a low polymer content, which ultimately reduce the physical strength of the core [1].

Additive manufacturing is the creation of a three-dimensional object from a computer-aided design (CAD) model by adding material layer by layer. AM systems for metallic particles can be classified into three main areas: powder bed systems, powder feed systems, and wire feed systems [3]. Powder bed fusion consists of selective laser melting (SLM) and direct metal-laser sintering (DMLS). These methods use a laser beam to scan over an evenly spread out powder bed, selectively melting portions of the powder. Once the laser sinters the beads, another layer will be spread on top. After the printing is complete, the resulting product is retrieved from the powder bed [4].

Binder jet printing works by applying an adhesive binder, either silicate or an aqueous-based, onto a layer of powder and then briefly heating it to partially cure. Once the previous layer of beads is together, another layer will be spread on top by raking powder across the work area. This process is completed until it forms into the desired shape. The final shape is then cured in a furnace at a fixed temperature for a varying amount of time to obtain the desired density. The longer the SMC is cured, the higher the density will be [1-3]. Powder bed fusion, on the other hand, is the main method of additive manufacturing in industrial settings for metallic materials.

Samples of iron powder coated with phosphorus were printed using the binder-jet printing process and the direct metal laser sintering process. The binder-jet samples were cured for one hour at $1500^{\circ} \mathrm{F}$ to burn off the binder, and then held at $2300^{\circ} \mathrm{F}$ for one hour to allow for densification. The DMLS printed samples were printed on a rotating bed printer which alters the 
angle of the laser by 60 degrees for each layer. The DMLS samples did not have a post-printing cure. The samples were cut into multiple sections, mounted in two-part epoxy, and left to harden for 12-24 hours. Once mounted, the samples were polished using silicon carbide discs of increasing grit fineness (120 grit, 240 grit, 400 grit, 600 grit, 800 grit, and 1200 grit) followed by diamond suspension solution ( 6 micron, 3 micron, and 1 micron). The silicon carbide polishing was executed manually, and the diamond polishing was conducted using an auto-polisher. The polished samples were placed in a vibropolisher for 24 hours in a 0.05 micron diamond suspension to ensure a mirror finish for microstructure analysis.

Binder-jet printed parts exhibited large interconnected pores and residual binder in concentrated areas caused by a shortened curing time. The samples were found to have porosity ranging from thirty to forty percent, primarily caused from binder burn off. When the binder burns away the iron particles only have limited area for diffusion during curing which results in low density of the overall part. The phosphorous coating, as well, began diffusing into the iron particles at approximately $800^{\circ} \mathrm{F}$ and completely burned off at $1200^{\circ} \mathrm{F}$. The DMLS parts exhibited a minor amount of micropores identified sporadically throughout the sample. The samples had a porosity of approximately 7\%. The laser melted the iron powdered with each pass which allowed for the particles to combine into a semi-solid piece. However, the phosphorous coating was burned away when the laser melted the particle.

Without the insulating coating, eddy current loss increases, which contributes to inefficiency, overheating, and failure. Since the cure time and temperature are variable, binder-jet printing is ideal for preserving the insulating coating of the SMC. However, DMLS is ideal for obtaining the desired density needed for SMC applications.

\section{References:}

[1] H. Shokrollahi, and K. Janghorban. Journal of Materials Processing Technology 189 (2007) p. 1-12.

[2] M. Lauda, et al. Journal of Magnetism and Magnetic Materials 411 (2016).

[3] Frazier, W.E. Journal of Material Engineering and Performance 23 (2014).

[4] S. Bremen, W. Meiners, and A. Diatlov. Laser Technik Journal 9 (2012).
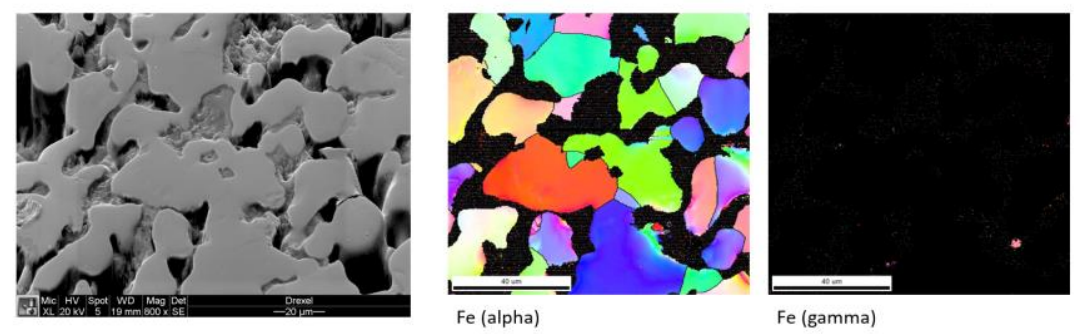

Figure 1. EBSD of binder-jet sample showing alpha and gamma regions of iron with high angle grain boundaries, some twin boundaries, and small angle grain boundaries. 